\title{
Acute fatal presentation of ornithine transcarbamylase deficiency in a previously healthy male
}

\author{
Ophir D. Klein · Dana R. Kostiner · Kara Weisiger · Ellen Moffatt · \\ Neal Lindeman · Stephen Goodman · Mendel Tuchman · Seymour Packman
}

Received: 4 October 2007/ Accepted: 3 April 2008/Published online: 7 May 2008

(C) The Author(s) 2008

\begin{abstract}
Ornithine transcarbamylase (OTC) deficiency is an X-linked urea cycle defect. While hemizygous males typically present with hyperammonemic coma in infancy, reports of rare late-onset presentations exist, with poor outcomes in males up to 58 years old. Relatives with mutations identical to affected patients often remain asymptomatic, and it is likely that environmental and genetic factors influence disease penetrance and expression. Here, we present our investigation of a patient with late-onset presentation, and we emphasize the potential role of environmental and genetic factors on disease
\end{abstract}

O. D. Klein $(\square)$

Departments of Orofacial Sciences and Pediatrics, and Institute of Human Genetics, University of California San Francisco, 513 Parnassus Ave., Box 0442, San Francisco, CA 94143, USA e-mail: ophir.klein@ucsf.edu

O. D. Klein · D. R. Kostiner · K. Weisiger · S. Packman Department of Pediatrics, Division of Medical Genetics, University of California San Francisco, San Francisco, CA, USA

Present Address:

D. R. Kostiner

Departments of Medical Genetics and Internal Medicine, Kaiser

Permanente Medical Center, San Francisco, USA

E. Moffatt $\cdot$ N. Lindeman

Department of Pathology, University of California San

Francisco, San Francisco, CA, USA

S. Goodman

University of Colorado School of Medicine/The Children's

Hospital, Denver, CO, USA

M. Tuchman

Children's Research Institute, Children's National Medical

Center, Washington, DC, USA expression. The patient was a previously healthy 62-yearold man who developed mental slowing, refractory seizures, and coma over an 8-day period. Interestingly, the patient had recently used home gardening fertilizers and pesticides. Evaluations for drug and alcohol use, infections, and liver disease were negative. Despite aggressive therapy, blood $\mathrm{NH}_{3}$ concentration peaked at $2,050 \mu \mathrm{M}$ and the patient died from cerebral edema and cerebellar herniation. Analysis of the OTC gene showed a Pro-225-Thr (P225T) change in exon 7 , a mutation that has been previously implicated in OTC deficiency. This case illustrates that OTC deficiency can cause acute, severe hyperammonemia in a previously healthy adult and that the P225T mutation can be associated with late-onset OTC deficiency. We speculate that exposure to organic chemicals might have contributed to the onset of symptoms in this patient. This case also emphasizes that persistent hyperammonemia may cause irreversible neurologic damage and that after the diagnosis of hyperammonemia is established in an acutely ill patient, certain diagnostic tests should be performed to differentiate between urea cycle disorders and other causes of hyperammonemic encephalopathy.

Keywords Hyperammonemia - OTC .

Ornithine transcarbamylase $\cdot$ Urea cycle $\cdot$ Late onset

\section{Introduction}

Ornithine transcarbamylase (OTC) deficiency (OMIM 311250), an X-linked disorder, is the most commonly inherited defect of the urea cycle. While hemizygous males typically present with hyperammonemic coma in the neonatal period, unusual cases have been reported with late-onset presentations in patients up to 58 years old, often 
with poor outcomes [1]. Subsequent mutation testing has shown that many relatives of these probands carry identical mutations but remain asymptomatic. It is likely that genetic and environmental factors exist that contribute to disease manifestations in some hemizygotes, while protecting others.

Here, we present the case of a previously healthy 62year-old man who developed mental slowing, refractory seizures, and coma over an 8-day period because of undiagnosed OTC deficiency. This case illustrates that urea cycle defects can present in adulthood and can be fatal. Acute neurologic decline and hyperammonemia in a previously healthy adult should be investigated and managed with this consideration in mind. Our investigation of this patient bears on the potential roles of genetic and environmental factors in the onset of disease expression.

\section{Methods}

Ammonia analysis

Free-flowing blood was collected by venipuncture into a lithium heparin tube placed on ice and was immediately analyzed by spectrophotometric and kinetic (glutamate dehydrogenase) means. No hemolysis was noted on any specimens and most were repeated to confirm initial measurements.

Orotic acid analysis

Urine specimen was collected and frozen for shipment to the laboratory at the Children's Hospital of Los Angeles. Stable isotope dilution was performed and the sample was analyzed by tandem mass spectrometry.

\section{OTC mutation analysis}

A sample from the patient's liver was flash frozen and sent to the University of Minnesota laboratory. Genomic DNA was extracted and PCR amplification of all 10 exons and exon/intron borders was performed. SSCP analysis was used to screen for abnormal mobility of the amplified gene fragments and sequencing of the abnormally migrating exons was performed.

\section{Blood gas analysis}

Standard blood gas analysis was performed on arterial blood.
Clinical diagnosis

The Glasgow coma scale, clinical observation of pupil dilation, arm and leg movements, cough and gag reflexes, and electroencephalogram for seizure activity were used to determine the clinical status of the patient. Cerebral edema and cerebellar herniation were observed on computed tomographic scan and brain magnetic resonance imaging and confirmed by autopsy.

\section{Case report}

A previously healthy, intelligent 62-year-old man developed mental slowing, visual changes, emesis, refractory seizures, and coma over an 8-day period. History and physical evaluation were negative for drug or alcohol use, infection, or liver disease. Of note, the patient had recently used several home-gardening products for the first time, including fertilizers, pesticides, and sealants, and he had dry cracked hands and did not wear gloves.

The initial blood ammonia level was elevated at $314 \mu \mathrm{g} /$ dl (normal range $[\mathrm{nl}]=17-80$ ) and rapidly rose to $2,050 \mu \mathrm{M}(\mathrm{nl}=11-35)$. The urine orotic acid level was markedly elevated at $473 \mathrm{mmol} / \mathrm{mol}$ creatinine $(\mathrm{nl}=0-3)$. The plasma citrulline level was normal at $18 \mu \mathrm{M} / \mathrm{L}$ $(\mathrm{nl}=12-55)$. Sodium benzoate, sodium phenylacetate, arginine free base, lactulose, intravenous glucose, and hemodialysis were initiated and the ammonia level gradually dropped to $218 \mu \mathrm{M}$. However, the patient developed cerebral edema and died because of cerebellar herniation, severe hypotension, and multisystem organ failure (confirmed by autopsy).

\section{Results}

Mutation analysis of the OTC gene showed a single base change (CCG $\rightarrow$ ACG) in exon 7, leading to the substitution of a threonine for a proline at position 225 (Pro-225Thr, P225T).

After learning of the proband's diagnosis, several family members underwent mutation analysis for the P225T substitution. One uncle, two brothers, and one great-nephew were found to be hemizygous for the mutation (Fig. 1). The six daughters of the hemizygous brothers were obligate heterozygotes for the mutation. None of these 10 family members, ranging in age from 2 to 96 years, has received pharmacologic or dietary treatment. They have remained asymptomatic with normal cognition and good health. The male relatives have had their orotic acid and ammonia levels checked, and these have been found to be within the normal range. 
Fig. 1 Pedigree of family presented in case report. Index patient is represented by black square

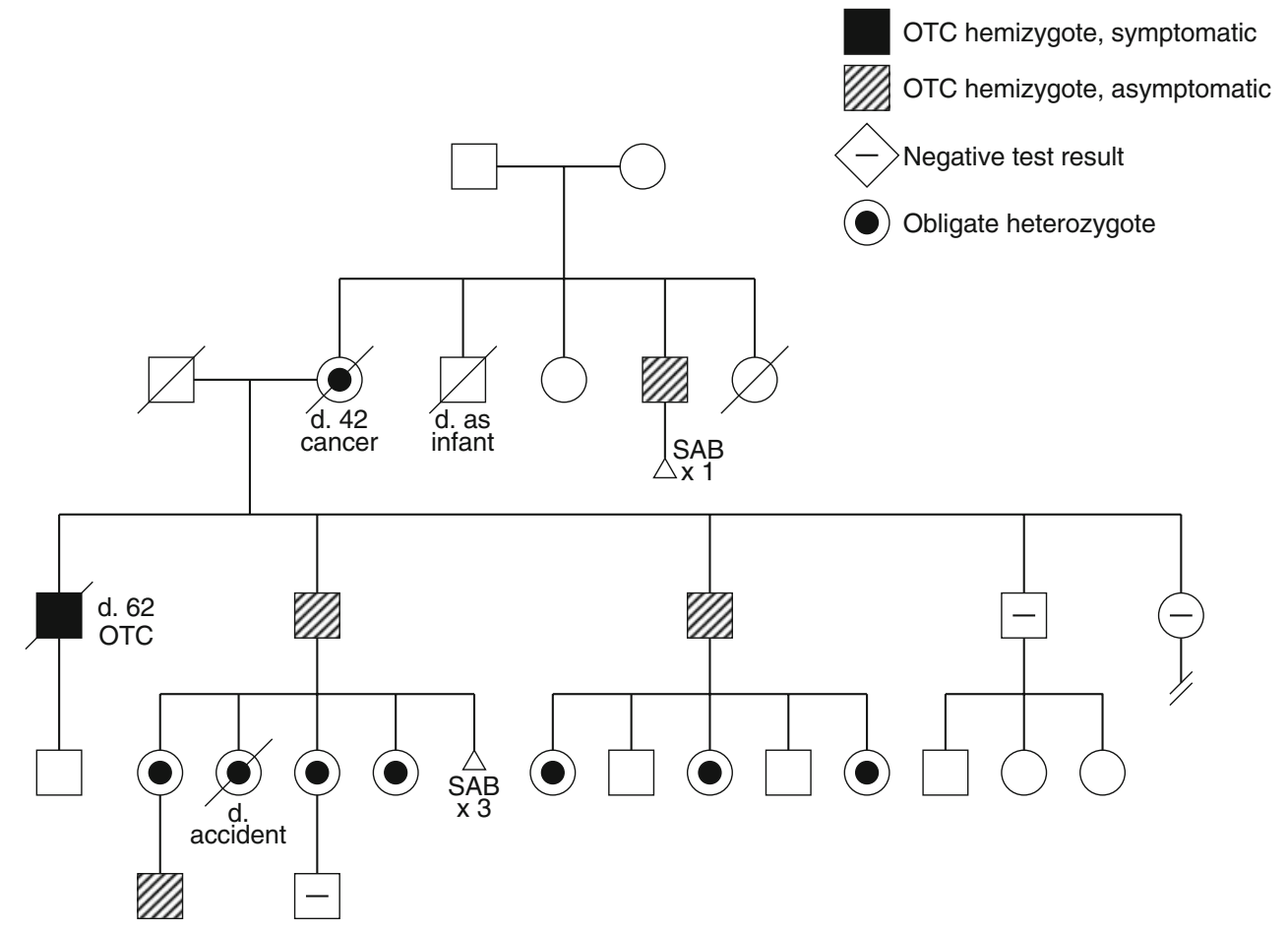

\section{Discussion}

More than 230 disease-causing OTC mutations have been identified, many of which are unique to the families in which they occur [2]. In general, mutations that affect amino acids in the interior of the enzyme near the active site-such as proline-225-cause neonatal disease, whereas mutations affecting residues on the surface of the protein are associated with milder phenotypes or later onset of disease [1]. However, in some cases, mutations that affect the catalytic function of the mutant enzyme are associated with late-onset disease. In particular, this report demonstrates that OTC deficiency resulting from the P225T mutation can cause such late-onset disease.

The proline-225 residue affected in this patient is conserved among many species and may be involved in the processing of the OTC protein [3]. Different amino acid substitutions at proline-225 can have substantially different disease presentations. For example, Pro-225-Arg [3] and Pro-225-Leu [4] have been found in males with severe neonatal OTC deficiency. The P225T mutation has been documented in one other patient, a previously healthy boy who manifested late-onset disease at 8 years of age [5]. In a stable isotope study of ureagenesis, this particular mutation resulted in a $42 \%$ reduction in ${ }^{15} \mathrm{~N}$ incorporation into urea, indicating that $\mathrm{P} 225 \mathrm{~T}$ allows for significant residual OTC activity [6].

A variety of genetic and environmental factors exist that can lead a previously healthy hemizygote to become acutely ill later in life. Some late-onset mutations, such as
$\mathrm{R} 40 \mathrm{H}$, have been shown to affect posttranslational processing events such as targeting to the mitochondrion [1]. Several environmental factors may also contribute to the timing of disease onset and disease manifestations. These factors include high-protein diets, valproic acid treatment, and exposure to the insect repellant $N, N$-diethyltoluamide [7-9]. Electron microscopic studies of the liver of a lateonset OTC patient revealed mitochondrial abnormalities beyond those expected with OTC deficiency per se, suggesting primary mitochondrial injury by exogenous agents [10]. Organophosphates, such as the pesticides to which this patient was exposed to, have been reported to induce apoptosis by disrupting mitochondrial membranes and activating caspases [11]. Our patient was in good health until 62 years of age, when he became acutely ill and died. In the days preceding his illness, he worked with several home-gardening products, including pesticides, fertilizers, and sealants. We speculate that exposure to organic chemicals may have contributed to the onset of symptoms in this patient; that is, chemicals may have entered his system through his lungs or skin, accumulated in his liver, and compromised his OTC function.

The differential diagnosis of hyperammonemia in an older child or adult can be addressed by routine laboratory testing. Causes of hyperammonemia include both nongenetic (acquired) and genetic etiologies, and include postviral aspirin-induced Reye syndrome; acute or chronic hepatitis; Wilson disease; $\alpha_{1}$-antitrypsin deficiency; alcoholic cirrhosis; drug-induced liver failure (e.g., anticonvulsants); chemotherapy; infection; and late-onset urea 
Table 1 Serial laboratory values of patient

\begin{tabular}{|c|c|c|c|c|c|c|c|c|}
\hline & Ammonia & $\mathrm{pH}$ & $\mathrm{pCO}_{2}$ & $\mathrm{HCO}_{3}$ & AST & ALT & Orotic acid & $\begin{array}{l}\text { Therapeutics for } \\
\text { hyperammonemia }\end{array}$ \\
\hline $\begin{array}{l}\text { Date/normal } \\
\text { range }\end{array}$ & $11-35 \mu \mathrm{mol} / 1$ & $7.35-7.45$ & $35-45$ & $21-27$ & $12-42 \mathrm{U} / 1$ & $9-50 \mathrm{U} / 1$ & $\begin{array}{l}0-3 \mathrm{mmol} / \mathrm{mol} \\
\text { creatinine }\end{array}$ & \\
\hline Day $1^{\mathrm{a}}$ & 314 & 7.47 & 33 & 24 & 22 & 33 & nd & Lactulose \\
\hline Day $4^{\mathrm{a}}$ & 203 & nd & nd & nd & nd & nd & nd & Lactulose \\
\hline Day $5^{\mathrm{a}}$ & 183 & 7.5 & 29 & 23 & 24 & 36 & nd & Lactulose \\
\hline Day 6, 0635 & 457 & 7.6 & 20.1 & 20 & 28 & 34 & nd & $\begin{array}{l}\text { Lactulose, Sodium Benzoate, } \\
\text { Phenylacetate }\end{array}$ \\
\hline Day 6,2150 & 1345 & 7.3 & 50.6 & 24.3 & nd & nd & nd & \\
\hline Day 7, 0330 & 1240 & 7.44 & 33.1 & 22.2 & nd & nd & nd & $\begin{array}{l}\text { Lactulose, Sodium Benzoate, } \\
\text { Phenylacetate, Arginine free base }\end{array}$ \\
\hline Day 7, 0930 & 2050 & 7.45 & 33 & 22.6 & nd & nd & nd & \\
\hline Day 7,1210 & 1269 & nd & nd & nd & nd & nd & 473 & CVVHD started \\
\hline Day 8,1151 & 218 & 7.14 & 29.5 & 9.5 & nd & nd & nd & $\begin{array}{l}\text { Lactulose, Sodium Benzoate, } \\
\text { Phenylacetate, Arginine free base }\end{array}$ \\
\hline
\end{tabular}

${ }^{a}$ Labs done at outside hospital prior to transfer; CVVHD-continuous veno-venous hemodialysis

nd-not done

cycle defects. In a patient with hyperammonemia, a complete blood cell (CBC) count, serum electrolytes (including measurement of the anion gap), blood $\mathrm{pH}$, blood glucose, liver function chemistries, and urinalysis can be helpful. In this case, a respiratory alkalosis was the only abnormality found initially on laboratory analysis (Table 1). Specifically, a normal anion gap existed, liver functions and CBC were normal, and blood glucose $(145 \mathrm{mg} / \mathrm{dl})$ and urinalysis (trace ketones) were near normal values. As a first approximation, hyperammonemia in the presence of normal liver function and a respiratory alkalosis is highly suggestive of a urea cycle defect. Additional tests are needed to determine which urea cycle defect is present, and this additional testing includes measurement of quantitative plasma amino acids and urine orotic acid.

Treatment guidelines for hyperammonemia include the following:

- Intravenous hydration with glucose and lipids to minimize protein catabolism and endogenous ammonia production.

- Cessation of protein intake from all sources to restrict nitrogen intake.

- Administration of arginine $\mathrm{HCl}$ or arginine free base for all urea cycle defects except arginase deficiency. This allows the urea cycle to partially proceed with removal of some fraction of accumulated nitrogen.

- Administration of compounds that facilitate the removal of ammonia through alternate pathways, including oral sodium phenylbutyrate, which conjugates glutamine, leading to the removal of two nitrogens as phenylacetylglutamine, and oral sodium benzoate, which conjugates glycine, leading to removal of 1 nitrogen as hippurate. These can be given orally or, in more severe presentations, combined in an intravenous formulation.

- Hemodialysis can be performed in severe cases marked by refractory hyperammonemia or encephalopathy, or when there is clinical deterioration.

- Treatment of shock, sepsis, seizures, and increased intracranial pressure.

The family members of the patient presented here raise an important clinical issue: when and whether to treat asymptomatic individuals with OTC mutations, given an index patient with late-onset disease [12]. In this case, we elected close clinical monitoring of the affected relatives, although a more aggressive approach may be warranted. Female carriers should be followed in addition to males.

Asymptomatic family members can opt for diet modification and prophylactic urea cycle defect therapy, but it is unclear whether these measures are necessarily more effective than close clinical monitoring in the prevention of hyperammonemia and metabolic decompensation.

Treatment possibilities for asymptomatic family members include:

- Carrying an emergency letter indicating that they are at risk of hyperammonemia. Included in the letter should be the laboratory testing to be obtained, the contact number of a metabolic specialist, and instructions for the dose and administration of arginine, sodium benzoate, and sodium phenylacetate.

- Monitoring of blood ammonia levels during intercurrent illnesses to determine whether ammonia levels are 
Table 2 Neurologic status of patient

\begin{tabular}{llllllll}
\hline & Pupils R/L & Glascow coma scale & Arm movement R/L & Leg movement R/L & Seizures & Gag reflex & Cough reflex \\
\hline Day 1 & a & Eyes $^{\mathrm{b}}$, Motor 5, Verbal 4 & Spontaneous & Spontaneous & None & Present & Present \\
Day 5 & $3 / 3.5$ brisk & Eyes 1, Motor 4, Verbal 1 & Flexion/extension & Trace voluntary & None & Present & Present \\
Day 6 & $5 / 5$ no rxn & Eyes 1, Motor 1, Verbal 1 & No movement & No movement & Present & None & None \\
Day 7 & $7.5 / 8$ no rxn & Eyes 1, Motor 1, Verbal 1 & No movement & No movement & Present & None & None \\
Day 8 & $4.5 / 4.5$ fixed & Eyes 1, Motor 1, Verbal 1 & No movement & No movement & None & None & None \\
\hline
\end{tabular}

${ }^{a}$ Outside hospital reported equal and reactive to light

b Preferred to keep eyes closed but would sometimes open to speech (3), usually only to pain (2)

${ }^{c}$ Endotracheal tube was placed on day 2

elevated during catabolic stress. If elevations occur, an emergency interval diet plan can be provided for fluid and nutritional requirements, including cessation of protein intake and provision of adequate calories.

- Ongoing dietary restriction of protein and supplementation with an essential amino acid formula.

In conclusion, it is important to recognize that acute neurologic decline and hyperammonemia in a previously healthy adult may be due to a urea cycle defect (Table 2). Vigilance by clinicians can lead to rapid treatment and appropriate involvement of metabolic specialists. Finally, molecular studies in patients such as the one in this report will help identify genotype-phenotype correlations and contribute to our understanding of disease mechanisms in this heterogenous disorder.

Acknowledgments Portions of this work were supported by NIH M01-RR01271 (to the UCSF Pediatric Clinical Research Center) and NIDDK R01-DK47870 (to M.T.). O.K. was supported by a Pediatric Scientist Development Program award from the NIH (K12HD00850). Finally, we thank the family of the patient presented in this report for their courage and understanding.

Open Access This article is distributed under the terms of the Creative Commons Attribution Noncommercial License which permits any noncommercial use, distribution, and reproduction in any medium, provided the original author(s) and source are credited.

\section{References}

1. Tuchman M, Morizono H, Rajagopal BS, Plante RJ, Allewell NM. The biochemical and molecular spectrum of ornithine transcarbamylase deficiency. J Inherit Metab Dis 1998;21 (Suppl 1):40-58.

2. Tuchman M, Jaleel N, Morizono H, Sheehy L, Lynch MG. Mutations and polymorphisms in the human ornithine transcarbamylase gene. Hum Mutat 2002;19:93-107.

3. Garcia-Perez MA, Climent C, Briones P, Vilaseca MA, Rodes M, Rubio V. Missense mutations in codon 225 of ornithine transcarbamylase (OTC) result in decreased amounts of OTC protein: a hypothesis on the molecular mechanism of the OTC deficiency. J Inherit Metab Dis 1997;20:769-77.

4. Hentzen D, Pelet A, Feldman D, Rabier D, Berthelot J, Munnich A. Fatal hyperammonemia resulting from a C-to-T mutation at a $M s p I$ site of the ornithine transcarbamylase gene. Hum Genet 1991;88:153-6.

5. Tuchman M, Plante RJ, McCann MT, Qureshi AA. Seven new mutations in the human ornithine transcarbamylase gene. Hum Mutat 1994;4:57-60.

6. McCullough BA, Yudkoff M, Batshaw ML, Wilson JM, Raper SE, Tuchman M. Genotype spectrum of ornithine transcarbamylase deficiency: correlation with the clinical and biochemical phenotype. Am J Med Genet 2000;93:313-9.

7. Gordon N. Ornithine transcarbamylase deficiency: a urea cycle defect. Eur J Paediatr Neurol 2003;7:115-21.

8. Gaspari R, Arcangeli A, Mensi S, Wismayer DS, Tartaglione T, Antuzzi D, Conti G, Proietti R. Late-onset presentation of ornithine transcarbamylase deficiency in a young woman with hyperammonemic coma. Ann Emerg Med 2003;41:104-9.

9. Heick HM, Peterson RG, Dalpe-Scott M, Qureshi IA. Insect repellent, N,N-diethyl-m-toluamide, effect on ammonia metabolism. Pediatrics 1988;82:373-6.

10. Tallan HH, Schaffner F, Taffet SL, Schneidman K, Gaull GE. Ornithine carbamoyltransferase deficiency in an adult male patient: significance of hepatic ultrastructure in clinical diagnosis. Pediatrics 1983;71:224-32.

11. Saleh AM, Vijayasarathy C, Masoud L, Kumar L, Shahin A, Kambal A. Paraoxon induces apoptosis in EL4 cells via activation of mitochondrial pathways. Toxicol Appl Pharmacol 2003;190:47-57.

12. Wilcken B. Problems in the management of urea cycle disorders. Mol Genet Metab 2004;81(Suppl):86-91. 